\title{
AP1- and NF-kB-binding sites conserved among mammalian WNT10B orthologs elucidate the TNF $\alpha$-WNT10B signaling loop implicated in carcinogenesis and adipogenesis
}

\author{
MASUKO KATOH ${ }^{1}$ and MASARU KATOH ${ }^{2}$ \\ ${ }^{1}$ M\&M Medical BioInformatics, Hongo 113-0033; ${ }^{2}$ Genetics and Cell Biology Section, \\ National Cancer Center Research Institute, Tokyo 104-0045, Japan \\ Received January 11, 2007; Accepted February 12, 2007
}

\begin{abstract}
WNT signals are context-dependently transduced to canonical and non-canonical signaling cascades. We cloned and characterized wild-type human WNT10B, while another group cloned aberrant human WNT10B with Gly60Asp aminoacid substitution. Proto-oncogene WNT10B is expressed in gastric cancer, pancreatic cancer, breast cancer, esophageal cancer, and cervical cancer. Because WNT10B blocks adipocyte differentiation, coding SNP of WNT1OB gene is associated with familial obesity. In 2001, we reported WNT10B upregulation by $\mathrm{TNF} \alpha$. Here, comparative integromics analyses on WNT10B orthologs were performed to elucidate the transcriptional mechanism of WNT10B. Chimpanzee WNT1OB and cow Wnt10b genes were identified within NW_001223159.1 and AC150975.2 genome sequences, respectively, by using bioinformatics (Techint) and human intelligence (Humint). Chimpanzee WNT10B and cow Wnt10b showed 98.7\% and $95.1 \%$ total-amino-acid identity with human WNT10B, respectively. N-terminal signal peptide, 24 Cys residues, two Asn-linked glycosylation sites, and Gly60 of human WNT10B were conserved among mammalian WNT10B orthologs. Transcription start site of human WNT10B gene was 106-bp upstream of NM_003394.2 RefSeq 5'-end. Number of GC dinucleotide repeats just down-stream of WNT1OB transcription start site varied among primates and human population. Comparative genomics analyses revealed that double AP1binding sites in the 5'-flanking promoter region and NF-кBbinding site in intron 3 were conserved among human, chimpanzee, cow, mouse, and rat WNT1OB orthologs. Because
\end{abstract}

Correspondence to: Dr Masaru Katoh, Genetics and Cell Biology Section, National Cancer Center Research Institute, 5-1-1 Tsukiji, Chuo-ku, Tokyo 104-0045, Japan

E-mail: mkatoh-kkr@umin.ac.jp

Key words: WNT, cytokine, tumor necrosis factor $\alpha$, Helicobacter pylori, hepatocellular carcinoma, diabetes mellitus, integrome network, systems medicine
TNF $\alpha$ signaling through TNFR1 and TRADD/RIP/TRAF2 complex activates JUN kinase (JNK) and IкB kinase (IKK) signaling cascades, conserved AP1- and NF- $\mathrm{KB}$-binding sites explain the mechanism of TNF $\alpha$-induced WNT10B upregulation. $\mathrm{TNF} \alpha-\mathrm{WNT} 10 \mathrm{~B}$ signaling loop is the negative feedback mechanism of adipogenesis to prevent obesity and metabolic syndrome. On the other hand, TNF $\alpha$-WNT10B signaling loop is implicated in carcinogenesis. Inhibitors of TNF $\alpha$-WNT10B signaling loop could be utilized for the prevention or treatment of cancer associated with chronic inflammation, such as gastric, liver, breast and pancreatic cancer.

\section{Introduction}

WNT family members with conserved 22 or 24 Cys residues are implicated in embryogenesis and adult tissue homeostasis (1-7). WNT signals are context-dependently transduced through Frizzled (FZD) receptors to canonical and non-canonical signaling cascades (8-11). Canonical WNT signal transduction leads to the upregulation of $M Y C, C C N D 1, F G F 20, J A G 1$, and $D K K 1$ genes for the cell fate determination (12-16), while non-canonical WNT signal transduction leads to the activation of RHOA, JUN kinase (JNK), PKC, NFAT and NLK signaling cascades for the regulation of tissue polarity, cell adhesion, and cell movement (17-19). Dysregulation of human WNT signaling cascades results in pathological conditions, such as cancer, rheumatoid arthritis, obesity, metabolic syndrome, and congestive heart failure (11).

We cloned and characterized wild-type human WNT10B (20), while another group cloned aberrant human WNT10B with Gly60Asp amino-acid substitution (21). We then identified and characterized rat Wnt10b (22). Human $W N T 10 B$ is expressed in a variety of cancer, such as gastric, pancreatic, breast, esophageal and cervical cancer $(20,23,24)$. Mouse Wnt $10 b$ upregulation due to the MMTV proviral integration results in the mammary carcinogenesis (25). Aberrant upregulation of WNT1OB leads to carcinogenesis through the canonical WNT signaling activation. WNTIOB is a proto-oncogene.

Mesenchymal stem cells give rise to a variety of cell lineages, such as adipocyte, osteocyte, chondrocyte, fibroblast, myocyte, and hepatocyte. WNT10B activates the canonical 


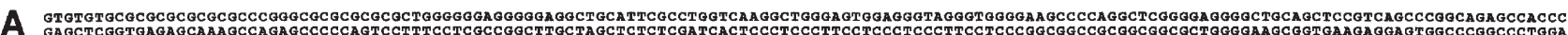

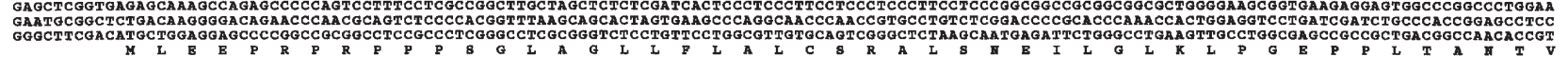

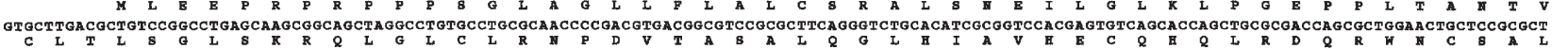

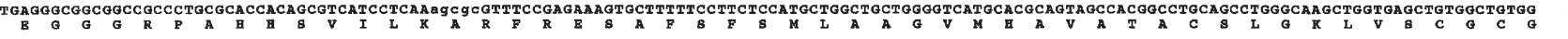

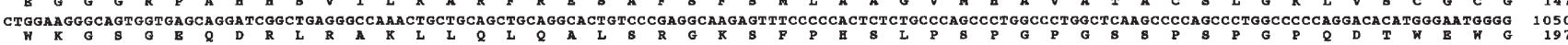

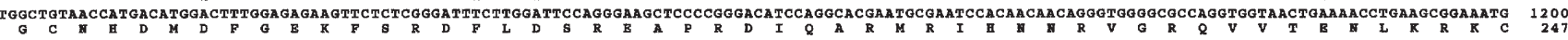
CAAGTGTCATGGCACGTCAGGCAGCTGCCAGTTCAAGACATGCTGGAGGGCGCCCCAGAGTTCCGGGCAGTGGGGCGGGCGTTGAGGaGCGGCTGGGCCGGGCCATCTTCATTGATACCCACAACCGCAATTCTGGAGCCTTCCAGCC 1350

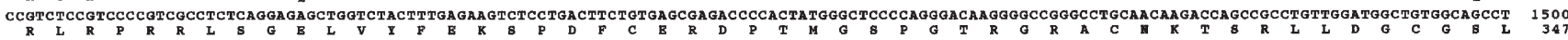

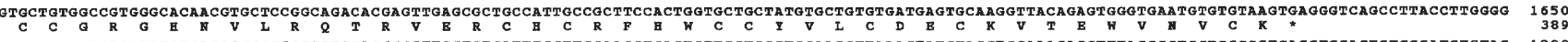

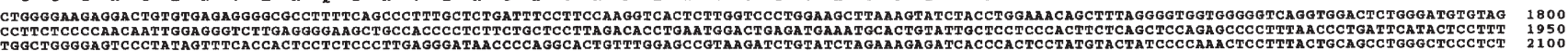

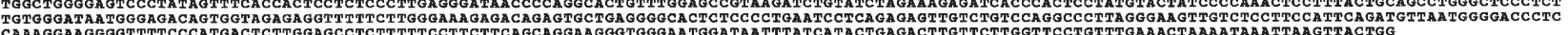

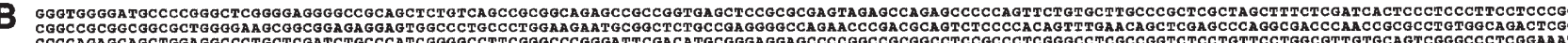

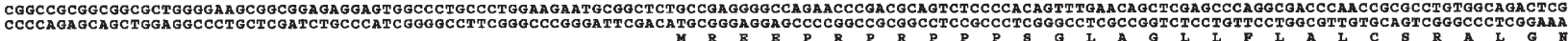

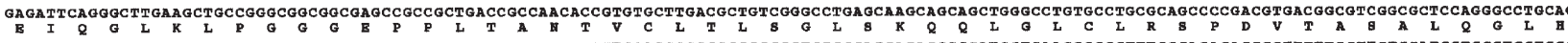

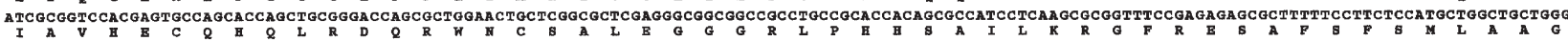

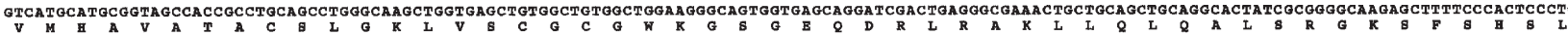

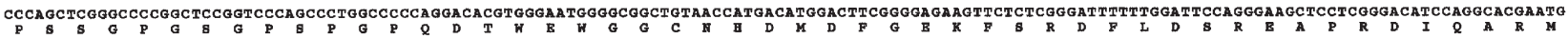

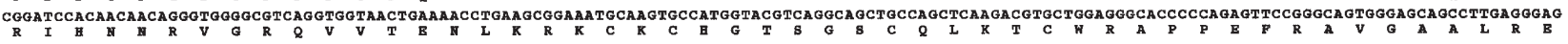

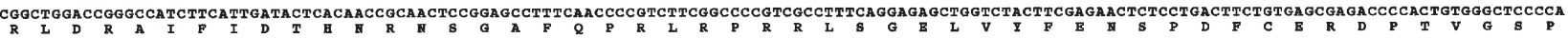

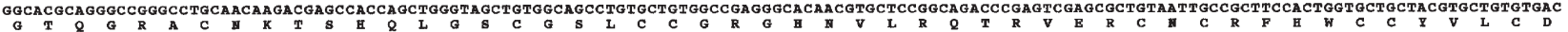
GAGTGCAaGgTCACAGAGTGGGTCAATGTGTGTAaGTG

GAAACAGCTTTGGGTCAGGTGGAGTCTGTGATGTGTGGCTCTCTCCCCACAGTAGGAGGGCCTTOTCAGGGAGCTGTCACCCTCTTCTGCTCCTTGACACCTGAATGAAGAAGATGAATGAACTGTGTTGCTCTCCAGCCACCCA

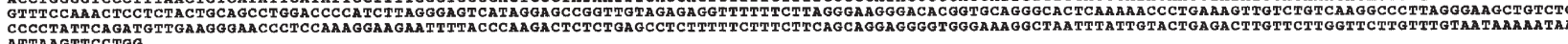
.

Figure 1. Chimpanzee and cow WNT10B orthologs. (A), Chimpanzee WNT10B complete CDS. (B), Cow Wnt10b complete CDS. Nucleotides and aminoacid residues are numbered on the right.

WNT signaling cascade to block adipocyte differentiation (26). Coding SNP of WNT10B gene to introduce missense mutation on one of conserved Cys residues is associated with familial obesity (27). Because loss-of-function coding SNP of WNT1OB leads to obesity, WNT1OB is a suppressor gene for metabolic syndrome.

In 2001, we reported upregulation of $W N T 1 O B$ by TNF $\alpha$ (20). TNF $\alpha$ signal is transduced through TNFR1 and TRADD/ RIP/TRAF2 complex to JNK and IкB kinase (IKK) signaling cascades, which leads to the activation of AP1 and NF-кB transcriptional complexes, respectively (28). Here, comparative integromics analyses on WNT10B orthologs were performed to elucidate the mechanism of $\mathrm{TNF} \alpha$-induced $W N T 10 B$ upregulation. Chimpanzee $W N T 10 B$ and cow Wnt10b genes were identified and characterized at first. AP1- and NF-кBbinding sites in the promoter and introns of WNT1OB orthologs were then searched for. Evolutionarily conserved AP1-binding sites in the 5'-flanking promoter region and NF-кB-binding site in intron 3 of mammalian WNT1OB orthologs were successfully identified in this study. Based on these facts, we propose that the $\mathrm{TNF} \alpha-\mathrm{WNT} 10 \mathrm{~B}$ signaling loop is implicated in carcinogenesis and adipogenesis.

\section{Materials and methods}

Identification and characterization of chimpanzee and cow WNT1OB orthologs. Chimpanzee and cow genome sequences homologous to human WNT1OB were searched for with BLAST programs as described previously (30-33). Exon-intron boundaries were determined based on the consensus sequence of exon-intron junctions ('gt ... ag' rule of intronic sequence) and codon usage within the coding region as described previously (34-37). Complete coding sequence (CDS) of chimpanzee WNT10B and cow Wnt10b were determined by assembling exonic regions.

Comparative proteomics analyses. Complete CDS of chimpanzee and cow WNT10B orthologs were translated into amino-acid sequence by using Genetyx program. Mammalian WNT10B orthologs were then aligned for the comparative integromics analyses.

Comparative genomics analyses. Human genome sequence around the WNTIOB gene was compared with chimpanzee, cow, mouse and rat genome sequences to identify evolutionarily conserved regions by using the BLAST programs. Transcription factor-binding sites within the evolutionarily conserved regions were then searched for by using Match program, Genetyx program, and the manual curation as described previously (38-41).

\section{Results}

Transcription start site of human WNT10B. BLAST programs using human WNT10B RefSeq (NM_003394.2) revealed that WNT1OB gene at human chromosome $12 \mathrm{q} 13$ was located within human genome sequence AC025256.33. BLAST programs using human genome sequence around the WNT10B gene next revealed that several WNT10B ESTs were transcribed from more upstream position than the 5'-end of NM_003394.2 RefSeq. DA359350.1 and DA530656.1 ESTs were transcribed from the 106-bp upstream position. Number of GC di-nucleotide repeats just down-stream of WNT10B transcription start site was 9 in DA359350.1 and DA530656.1 ESTs, but was 11 in AC025256.33 genome sequence. Based on these facts, it was concluded that the transcription start site of 


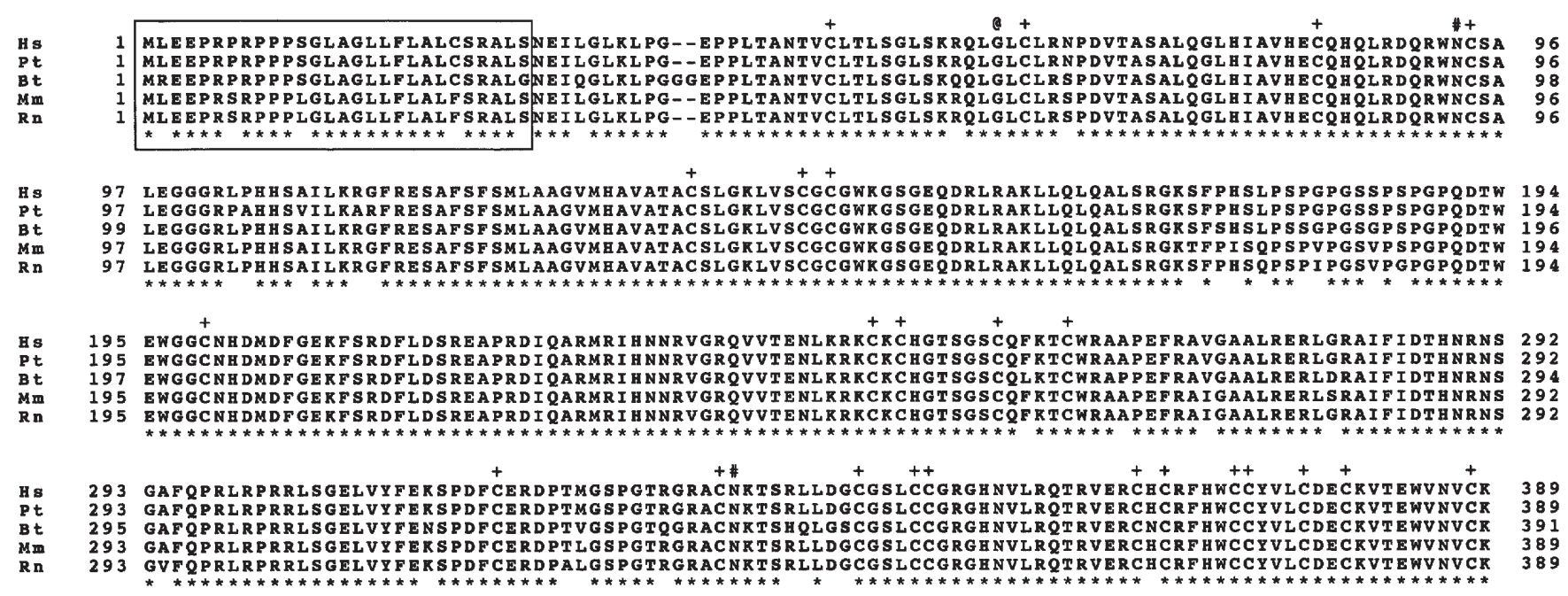

Figure 2. Mammalian WNT10B orthologs. Hs, human; Pt, chimpanzee; Bt, cow; Mm, mouse; Rn, rat. Amino-acid residues are numbered on the right. Signal peptide is boxed. Conserved Cys residues (cross) and Asn-linked glycosylation sites (sharp) are shown above the alignment. Location of Gly60Asp aminoacid substitution (@) is also shown. Gly60 of wild-type human WNT10B cloned by us (20) is conserved among mammalian WNT10B orthologs.

human WNT10B gene was 106-bp upstream of NM_003394.2 RefSeq 5'-end.

Chimpanzee WNT10B and cow Wnt10b genes. BLAST programs using human WNT10B complete CDS revealed that chimpanzee WNT1OB gene was located within NW_001223159.1 genome sequence. Exon-intron boundaries of chimpanzee $W N T 10 B$ gene were determined based on the consensus sequence of exon-intron junctions. Chimpanzee WNT10B gene was found consisting of five exons. Complete CDS of chimpanzee WNT10B was determined by assembling exonic regions (Fig. 1A). Number of GC di-nucleotide repeats just down-stream of WNT1OB transcription start site was 15 in chimpanzee NW_001223159.1 genome sequence. Genetyx program revealed that nucleotide position 461-1630 was the coding region. Chimpanzee WNT1OB gene was found to encode a 389-amino-acid protein (Fig. 1A).

BLAST programs next revealed that cow Wnt $10 b$ gene was located within AC150975.2 genome sequence. Complete CDS of cow Wnt10b was determined by assembling five exonic regions (Fig. 1B). Genetyx program revealed that nucleotide position 364-1539 was the coding region. Cow $W n t 10 b$ gene was found to encode a 391-amino-acid protein (Fig. 1B).

Comparative proteomics analyses on WNT10B orthologs. Chimpanzee WNT10B and cow Wnt10b showed 98.7\% and $95.1 \%$ total-amino-acid identity with human WNT10B, respectively. $\mathrm{N}$-terminal signal peptide, $24 \mathrm{Cys}$ residues and two Asn-linked glycosylation sites were conserved among human, chimpanzee, cow, mouse, and rat WNT10B orthologs. In addition, Gly60 rather than Asp60 of human WNT10B were conserved among mammalian WNT10B orthologs (Fig. 2).

Comparative genomics analyses on WNT10B orthologs. Human WNT1OB, chimpanzee WNT1OB and cow Wnt10b genes are located within AC025256.33, NW_001223159.1 and AC150975.2 genome sequences, respectively, as mentioned above. Rat Wnt10b gene is located within AC118760.4 genome sequence as reported previously (22). BLAST programs using mouse Wnt10b RefSeq (NM_011718.1) revealed that mouse Wnt10b gene was located within AC156543.10 genome sequence.

Conserved regions around the WNT1OB gene were next searched for. BLAST programs revealed that the 5'-flanking promoter region, five exonic regions, and intron 3 were well conserved between human $W N T 10 B$ and mouse Wnt $10 b$ genes (Fig. 3A). Transcription factor-binding sites within the 5'flanking promoter region and intron 3 were next searched for. Double AP1-binding sites located at about 2.2- and 0.9-kb upstream of the transcriptional start site of human WNT1OB gene were conserved in chimpanzee, cow, mouse, and rat WNT10B orthologs (Fig. 3A). In addition, NF-кB-binding site in intron 3 of human $W N T 10 B$ gene was conserved in chimpanzee, cow, mouse, and rat WNT1OB orthologs (Fig. 3A).

\section{Discussion}

Comparative integromics analyses on WNT10B orthologs were performed. Chimpanzee WNTIOB gene was identified within NW_001223159.1 genome sequence (Fig. 1A), while cow Wnt10b gene within AC150975.2 genome sequence (Fig. 1B). Chimpanzee WNT10B and cow Wnt10b showed $98.7 \%$ and $95.1 \%$ total-amino-acid identity with human WNT10B, respectively. N-terminal signal peptide, 24 Cys residues, two Asn-linked glycosylation sites, and Gly60 of human WNT10B were conserved among mammalian WNT10B orthologs (Fig. 2).

Transcription start site of human WNT10B gene was located at 106-bp upstream of NM_003394.2 RefSeq 5'-end. GC dinucleotide repeats just down-stream of the transcriptional start site was identified in human and chimpanzee WNT1OB genes, but not in cow, mouse, and rat Wnt $10 b$ gene. Number of GC di-nucleotide repeats just down-stream of WNT10B transcription start site was 9 in human WNT10B ESTs DA359350.1 and DA530656.1, 11 in human genome sequence AC025256.33, and 15 in chimpanzee genome sequence 


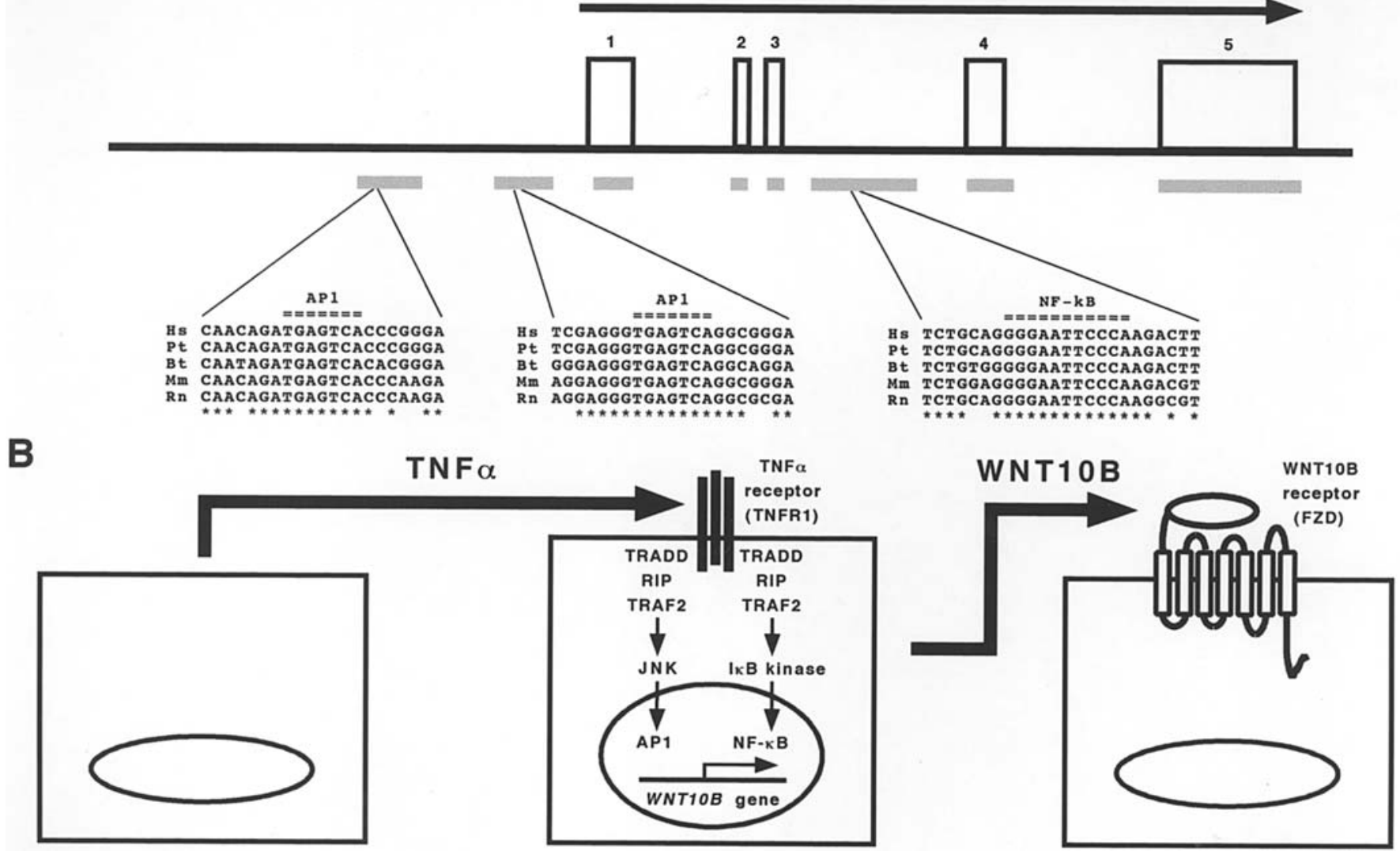

Figure 3. The TNF $\alpha$-WNT10B signaling loop. (A), AP1- and NF-kB-binding sites within mammalian WNT10B orthologs. Human WNT10B gene consists of five exons. Regions conserved between human $W N T 10 B$ and mouse $W n t 10 b$ genes are shown by gray bars. Hs, human; Pt, chimpanzee; Bt, cow; Mm, mouse; $\mathrm{Rn}$, rat. Double AP1-binding sites in the 5'-flanking promoter region and NF-kB-binding site in intron 3 are conserved among human, chimpanzee, cow, mouse, and rat $W N T 1 O B$ orthologs. WNTIOB gene is the evolutionarily conserved target of TNF $\alpha$ signaling cascade. (B), Schematic presentation of the TNF $\alpha-$ WNT10B signaling loop. TNFa-WNT10B signaling loop is the negative feedback mechanism of adipogenesis to prevent obesity and metabolic syndrome. On the other hand, TNFa-WNT10B signaling loop is implicated in the carcinogenesis associated with chronic inflammation

NW_001223159.1. These facts indicate that the number of GC di-nucleotide repeats just down-stream of WNT1OB transcription start site varied among primates as well as among human population.

We previously reported $W N T 10 B$ upregulation by $\mathrm{TNF} \alpha$ (20); however, the mechanism of TNF $\alpha$-induced WNT10B upregulation remained unclear. TNF $\alpha$ signaling through TNFR1 and TRADD/RIP/TRAF2 complex to JNK and IKK signaling cascades results in the activation of $\mathrm{AP} 1$ and NF- $\mathrm{\kappa B}$ transcriptional complex, respectively (28). In this study, we identified that double AP1-binding sites in the 5'-flanking promoter region and $\mathrm{NF}-\kappa \mathrm{B}-$ binding site in intron 3 were conserved among human, chimpanzee, cow, mouse, and rat WNT10B orthologs (Fig. 3A). Based on these facts, it was demonstrated that TNF $\alpha$ signaling to AP1 and NF- $\mathrm{KB}$ nuclear complex induces transcriptional upregulation of WNT1OB (Fig. 3B).

TNF $\alpha$ is secreted from mature adipocytes, and WNT10B blocks adipocytic differentiation. Because WNT1OB is a target gene of TNF $\alpha$ signaling cascade (Fig. 3), it is clear that the TNF $\alpha$-WNT10B signaling loop functions as the negative feedback loop to maintain the homeostasis of adipose tissue. Therefore, inactivation of the TNF $\alpha$-WNT10B signaling loop leads to obesity and metabolic syndrome.
Helicobacter pylori is a causative agent for peptic ulcer diseases, chronic gastritis, and gastric cancer (42-45). TNF $\alpha$ concentration is significantly elevated in patients with active gastritis than in those with inactive gastritis, while interleukin-6 is elevated in those with active and inactive gastritis (46). HBV and $\mathrm{HCV}$ are causative agents for hepatitis, liver cirrhosis, and liver cancer, and $\mathrm{TNF} \alpha$ concentration is also elevated during hepatocarcinogenesis (28). TNF $\alpha$ then induces upregulation of $W N T 10 B$ proto-oncogene during chronic inflammation (Fig. 3B). Because the TNF $\alpha$-WNT10B signaling loop plays a key role during carcinogenesis, inhibitors of the TNF $\alpha$ WNT10B signaling loop could be utilized for the prevention or treatment of cancer associated with chronic inflammation, such as gastric, liver, breast and pancreatic cancer.

\section{References}

1. Katoh M: WNT and FGF gene clusters. Int J Oncol 21: 1269-1273, 2002.

2. Katoh M: Regulation of WNT signaling molecules by retinoic acid during neuronal differentiation in NT2 cells: threshold model of WNT action. Int J Mol Med 10: 683-687, 2002.

3. Heller RS, Klein T, Ling Z, Heimberg H, Katoh M, Madsen OD and Serup P: Expression of WNT, Frizzled, sFRP, and DKK genes in adult human pancreas. Gene Expr 11: 141-147, 2003. 
4. Garciadiego-Cazares D, Rosales C, Katoh M and ChimalMonroy J: Coordination of chondrocyte differentiation and joint formation by $\alpha 5 \beta 1$ integrin in the developing appendicular skeleton. Development 131: 4735-4742, 2004.

5. Clevers H: Stem cells, asymmetric division and cancer. Nat Genet 37: 1027-1028, 2005.

6. Katoh M and Katoh M: Bioinformatics for cancer management in the post-genome era. Technol Cancer Res Treat 5: 169-176, 2006.

7. Katoh M and Katoh M: Cross-talk of WNT and FGF signaling pathways at GSK3ß to regulate $\beta$-catenin and SNAIL signaling cascades. Cancer Biol Ther 5: 1059-1064, 2006.

8. Swain RK, Katoh M, Medina A and Steinbeisser H: Xenopus frizzled-4S, a splicing variant of Xfz4, is a context-dependent activator and inhibitor of Wnt/ß-catenin signaling. Cell Commun Signal 3: 12, 2005.

9. Katoh M: WNT2B: comparative integromics and clinical application. Int J Mol Med 16: 1103-1108, 2005.

10. Katoh M: Epithelial-mesenchymal transition in gastric cancer. Int J Oncol 27: 1677-1683, 2005.

11. Katoh M and Katoh M: STAT3-induced WNT5A signaling loop in embryonic stem cells, adult normal tissues, chronic persistent inflammation, rheumatoid arthritis, and cancer. Int J Mol Med 19: 273-278, 2007

12. Katoh $\mathrm{M}$ and Katoh $\mathrm{M}$ : Identification and characterization of human $B C L 9 L$ gene and mouse Bcl9l gene in silico. Int $\mathrm{J}$ Mol Med 12: 643-649, 2003.

13. Chamorro MN, Schwartz DR, Vonica A, et al: FGF20 and DKK1 are transcriptional target of $B$-catenin and FGF20 is implicated in cancer and development. EMBO J 24: 73-84, 2005.

14. Katoh M and Katoh M: Comparative genomics on FGF2O orthologs. Oncol Rep 14: 287-290, 2005.

15. Katoh Y and Katoh M: Comparative genomics on $D K K 1$ orthologs. Int J Oncol 27: 275-279, 2005

16. Katoh M and Katoh M: Notch ligand, JAG1, is evolutionarily conserved target of canonical WNT signaling pathway in progenitor cells. Int J Mol Med 17: 681-685, 2006.

17. Katoh M: WNT/PCP signaling pathway and human cancer. Oncol Rep 14: 1583-1588, 2005.

18. Boutros M, Paricio N, Strutt DI, et al: Dishevelled activates JNK and discriminates between JNK pathways in planar polarity and wingless signaling. Cell 94: 109-118, 1998.

19. Dejmek J, Safholm A, Kamp Nielsen C, et al: Wnt-5a/Ca ${ }^{2+}$ induced NFAT activity is counteracted by Wnt-5a/Yes-Cdc42casein kinase Ia signaling in human mammary epithelial cells. Mol Cell Biol 26: 6024-6036, 2006.

20. Saitoh T, Kirikoshi H, Mine T and Katoh M: Proto-oncogene $W N T 10 B$ is upregulated by tumor necrosis factor $\alpha$ in human gastric cancer cell line MKN45. Int J Oncol 19: 1187-1192, 2001.

21. Hardiman G, Kastelein RA and Bazan JF: Isolation, characterization and chromosomal localization of human WNT10B. Cytogenet Cell Genet 77: 278-282, 1997.

22. Katoh $\mathrm{Y}$ and Katoh M: Identification and characterization of rat Wnt1 and Wnt10b genes in silico. Int J Oncol 26: 841-845, 2005.

23. Bui TD, Rankin J, Smith K, et al: A novel human Wnt gene, $W N T 10 B$, maps to $12 \mathrm{q} 13$ and is expressed in human breast carcinomas. Oncogene 14: 1249-1253, 1997.

24. Kirikoshi $\mathrm{H}$ and Katoh M: Expression and regulation of WNT1OB in human cancer: upregulation of WNT1OB in MCF-7 cells by B-estradiol and downregulation of WNT1OB in NT2 cells by retinoic acid. Int J Mol Med 10: 507-511, 2002.

25. Lee FS, Lane TF, Kuo A, et al: Insertional mutagenesis identifies a member of the Wnt gene family as a candidate oncogene in the mammary epithelium of int-2/Fgf-3 transgenic mice. Proc Natl Acad Sci USA 92: 2268-2272, 1995.
26. Ross SE, Hemati N, Longo KA, et al: Inhibition of adipogenesis by Wnt signaling. Science 289: 950-953, 2000.

27. Christodoulides C, Scarda A, Granzotto M, et al: WNT10B mutations in human obesity. Diabetologia 49: 678-684, 2006.

28. Karin M: Nuclear factor-кB in cancer development and progression. Nature 441: 431-436, 2006.

29. Katoh M: Paradigm shift in gene-finding method: from benchtop approach to desk-top approach. Int J Mol Med 10: 677-682, 2002.

30. Katoh $\mathrm{M}$ and Katoh $\mathrm{M}$ : Identification and characterization of human PRICKLE1 and PRICKLE2 genes as well as mouse Prickle1 and Prickle2 genes homologous to Drosophila tissue polarity gene prickle. Int J Mol Med 11: 249-256, 2003.

31. Katoh $\mathrm{M}$ and Katoh $\mathrm{M}$ : Identification and characterization of human DAPPER1 and DAPPER2 genes in silico. Int J Oncol 22: 907-913, 2003.

32. Katoh $\mathrm{M}$ and Katoh $\mathrm{M}$ : Identification and characterization of human FMNL1, FMNL2 and FMNL3 genes in silico. Int J Oncol 22: $1161-1168,2003$.

33. Katoh $\mathrm{M}$ and Katoh $\mathrm{M}$ : Identification and characterization of human HES2, HES3, and HES5 genes in silico. Int J Oncol 25: 529-534, 2004.

34. Katoh M and Katoh M: Identification and characterization of human HESL, rat Hesl and rainbow trout hesl genes in silico. Int J Mol Med 14: 747-751, 2005.

35. Katoh M: Molecular evolution of WNT2B orthologs. Int J Oncol 26: 1135-1139, 2005.

36. Katoh Y and Katoh M: WNT antagonist, SFRP1, is Hedgehog signaling target. Int J Mol Med 17: 171-175, 2006.

37. Katoh $\mathrm{Y}$ and Katoh M: FGF signaling inhibitor, SPRY4, is evolutionarily conserved target of WNT signaling pathway in progenitor cells. Int J Mol Med 17: 529-532, 2006.

38. Katoh M and Katoh M: CER1 is a common target of WNT and NODAL signaling pathways in human embryonic stem cells. Int J Mol Med 17: 795-799, 2006.

39. Katoh $\mathrm{M}$ and Katoh M: WNT antagonist, DKK2, is a Notch signaling target in intestinal stem cells: augmentation of negative regulation system for canonical WNT signaling pathway by Notch-DKK2 signaling loop in primates. Int J Mol Med 19: 197-201, 2007.

40. Katoh Y and Katoh M: Conserved POU-binding site linked to SP1-binding site within FZD5 promoter: transcriptional mechanisms of FZD5 in undifferentiated human ES cells, fetal liver/spleen, adult colon, pancreatic islet, and diffuse-type gastric cancer. Int J Oncol 30: 751-755, 2007.

41. Katoh M and Katoh M: Comparative integromics on FZD7 orthologs: conserved binding sites for PU.1, SP1, CCAAT-box and $\mathrm{TCF} / \mathrm{LEF} / \mathrm{SOX}$ transcription factors within 5'-promoter region of mammalian FZD7 orthologs. Int J Mol Med 19: 529-533, 2007.

42. Marshall BJ and Warren JR: Unidentified curved bacilli in the stomach of patients with gastritis and peptic ulceration. Lancet 1: 1311-1315, 1984

43. Katoh $\mathrm{Y}$ and Katoh M: Hedgehog signaling in gastric cancer. Cancer Biol Ther 4: 1050-1054, 2005.

44. Peek RM Jr and Crabtree JE: Helicobacter pylori infection and gastric neoplasia. J Pathol 208: 233-248, 2006.

45. Katoh M and Katoh M: FGF signaling network in the gastrointestinal tract. Int J Oncol 29: 163-168, 2006.

46. Crabtree JE, Shallcross TM, Heatley RV and Wyatt JI: Mucosal tumour necrosis factor alpha and interleukin-6 in patients with Helicobacter pylori associated gastritis. Gut 32: 1473-1477, 1991. 